\title{
A Reversed-Phase Intercalator Column for High Performance Liquid Chromatographic Separation of Oligonucleotides
}

\author{
Kunihiko Dohtsu, Kiyoshi Ohmori, Ryuji Fukuda, Shigeori Takenaka and Makoto Takagi \\ Department of Organic Synthesis, Faculty of Engineering, Kyushu University, Hakozaki, Fukuoka 812
}

\begin{abstract}
The use of intercalator-nucleic base stacking interactions is developed and experimentally examined for the separation of mononucleotides and oligonucleotides by high performance liquid chromatography (HPLC). Silica gel chromatographic supports were modified with anthracene- and phthalimide-containing groups. The chromatographic supports so obtained were used for the separation of mononucleotides and pentadecamer oligonucleotides under reversed-phase, isocratic conditions. The separation behavior was studied in comparison with that using standard octadecylsilyl-modified silica gel (ODS) support. Separation among nucleotides was quite different for intercalator-based supports and for the ODS support, suggesting the importance of stacking interactions of anthracene and phthalimide groups with nucleic bases. Directions for the development of HPLC supports based on such interactions are discussed.
\end{abstract}

Keywords Intercalator, oligonucleotide, mononucleotide, high performance liquid chromatography, sequencedependent separation

The importance of separation and determination of oligonucleotides is increasing in gene technology and related fields. Extensive efforts have been made for the development of liquid chromatography to achieve the separation between sequence isomers or oligonucleotides of similar molecular sizes. Since oligonucleotides are essentially polymeric anions (poly(phosphate diesters)) which contain hydrophobic moieties (nucleic bases), both anion-exchange and reversedphase chromatography can be used for their separation. While reversed-phase liquid chromatography, typically using ODS-type silica gel supports, is now widely used ${ }^{1}$, various mixed-mode chromatographies have been studied using benzoylated or naphthoylated DEAE-cellulose ${ }^{2}$, RPC-5 (Teflon powder coated with hydrophobic quaternary ammonium salt) ${ }^{3}$, and others. ${ }^{4,5}$

With ODS-type supports, oligonucleotides are supposed to be eluted in the order of their hydrophobicity. However, there is no clear way of describing or predicting the hydrophobicity of oligonucleotides in terms of nucleic base contents or in terms of base sequences of particular analyte oligonucleotides. Therefore, the separation conditions on ODS supports are rather empirical, not giving any general directions to the aimed separation nor allowing predictions of the ordering of elutions of oligonucleotides of similar molecular size. To increase the choices and to further improve the separation in chromatography of oligonucleotides, we should take advantage of physicochemical properties of oligonucleotides other than their hydrophobic and polyelectrolytic characteristics.

Intercalation is a unique interaction of some planar polyaromatic compounds with double-strand DNAs. ${ }^{6}$ A similar interaction is observed with (single-strand) oligonucleotides (pseudo-intercalation) ${ }^{7}$, and we have been exploring such interactions for use in chromatographic separation of oligonucleotides. ${ }^{8,9}$ Some intercalators show GC- or AT-base pair selectivity. ${ }^{10}$ Some even show base-sequence selectivity (e.g., selectivity to $3^{\prime}-\mathrm{GC}-5^{\prime}$ rather than $3^{\prime}-\mathrm{CG}-5^{\prime}$ ) to a dinucleotide (nucleotide dimer) unit. ${ }^{11}$ Therefore, one can think of a new chromatographic separation mode different from traditional reversed-phase and ionexchange modes, where the oligonucleotide separation based on the nucleic base content or on the frequency of occurrence of particular base sequence should become possible. We have recently studied the electrophoresis of oligonucleotides in the presence of intercalators and have confirmed such unique features of separation. In the present study, intercalatorloaded, reversed-phase HPLC supports were prepared and tested for the separation of mononucleotides and 15-mer oligonucleotides, as a preliminary exploration of the intended intercalator-based HPLC.

\section{Experimental}

\section{Apparatus}

A Shimadzu Model LC-5A liquid chromatograph 
which employed a Rheodyne Model 7125 sampling valve with a $20 \mu \mathrm{l}$ loop and a Union-Giken Model MCPD-350PC detector system were used throughout the study of mononucleotides. A Tosoh Model CCPM liquid chromatograph with a Rheodyne Model 7125 sampling valve with a $100 \mu$ l loop, a Tosoh Model UV8 model II spectrophotometer and a Graphtec Model SR 6211 recorder were used throughout the study of oligonucleotides.

\section{Materials}

3-Aminopropyl-modified silica gel was obtained from Tosoh Co. Ltd. (silica gel: diameter, $10 \mu \mathrm{m}$; micro pore, $100 \AA$; aminopropyl content, $0.9 \mathrm{mmol} / \mathrm{g}$ modified silica gel). The ODS-type reversed-phase column used was a TSKgel ODS-120T $(15 \mathrm{~cm} \times 4.6 \mathrm{~mm}$ i.d.) from Tosoh Co. 3'-AMP, 3'-CMP, 3'-GMP, and 3'-UMP were purchased from Yamasa Co. Ltd. 15-mer oligonucleotides were synthesized by using DNA synthesizer and used after conventional purification on HPLC.

Aminopropyl-silica gel was further modified with 9anthracenecarbonyl chloride and phthalic anhydride according to the following procedure.

Two grams of aminopropyl-silica gel were suspended in $30 \mathrm{ml}$ of chloroform. After the addition of $1.0 \mathrm{~g}$ (4.3 mmol) of 9-anthracenecarbonyl chloride, the mixture was refluxed for one day. The gel was filtered off and washed with chloroform and methanol. Elemental analysis indicated that about 35\% of the aminopropyl groups in the gel were modified with 9-anthracenecarbonyl groups.

Two grams of aminopropyl-silica gel was suspended in $50 \mathrm{ml}$ pyridine. After the addition of $2.0 \mathrm{~g}(13.6$ $\mathrm{mmol}$ ) of phthalic anhydride, the mixture was refluxed for one day. The gel was filtered off and washed successively with pyridine, chloroform and methanol. Elemental analysis indicated that all of the aminopropyl groups in the gel were converted to phthalimide derivatives.

The modified silica gel supports obtained above were packed into a $10 \mathrm{~cm} \times 4.0 \mathrm{~mm}$ i.d. stainless steel column by standard slurry procedures and used for HPLC.

\section{Procedures}

In the chromatography of mononucleotides, the mobile phase consisted of a mixture of aqueous phosphate buffer $(5.0 \mathrm{mM}, \mathrm{pH} \mathrm{6.86)}$ and acetonitrile. For oligonucleotides, a mixture of aqueous triethylamine acetate (TEAA) buffer (0.1 M, pH 7.01) and acetonitrile was used. The composition of the mixed solvents is expressed in volume percent $(v / v, Y \%)$ of organic solvent, i.e., $Y \mathrm{ml}$ of the organic solvent was mixed with $(100-Y) \mathrm{ml}$ of aqueous solution. The detector was operated at $260 \mathrm{~nm}$. A flow rate of $0.5 \mathrm{ml}$ $\mathrm{min}^{-1}$ was used. All experiments were carried out at ambient temperature $\left(24-26^{\circ} \mathrm{C}\right)$ under isocratic conditions.

\section{Results and Discussion}

\section{Chromatography of mononucleotides}

The structure of modified-silica chromatographic supports is schematically shown in Fig. 1. The effect of organic modifier (acetonitrile) in the eluent was examined in $5 \mathrm{mM}$ aqueous phosphate buffer system at $\mathrm{pH}$ 6.86. While such $\mathrm{pH}$ conditions prevent protonation or deprotonation on nucleic bases, the aminopropyl residues which remained intact on reaction with acid chloride or acid anhydride are most likely protonated. This would give anthracene- and phthalimide-type columns ion exchange character and, associated with it, also hydrophilic character.

Figure 2 shows the effect of acetonitrile on the capacity factor of three mononucleotides on the ODS column. It is seen that an increase in acetonitrile content causes a general decrease in capacity factor, the behavior being typical of a reversed-phase chromatographic system. The effect is more pronounced with AMP than with GMP and CMP. These results can be explained on the basis of the hydrophobicity of

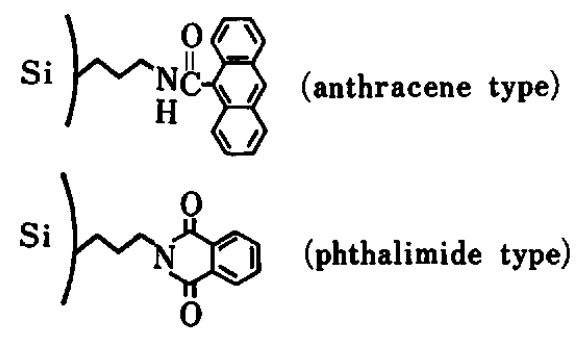

Fig. 1 Structure of modified-silica chromatographic supports.

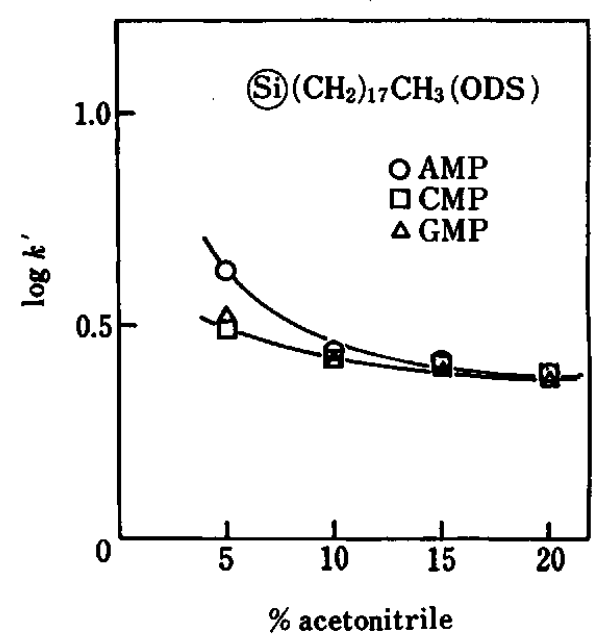

Fig. 2 Chromatography of mononucleotides on ODS support. Dependence of capacity factor $\left(k^{\prime}\right)$ on organic modifier (acetonitrile) content in mobile phase. $k^{\prime}=\left(v_{\mathrm{R}}-v_{0}\right) / v_{0}\left(v_{\mathrm{R}}\right.$, retention volume of sample; $v_{0}$, dead volume). 
nucleic bases. The strongest retention of AMP is due to the fact that the hydrophobicity of AMP is stronger than that of other nucleic bases. Interestingly, it was found that the distribution ratio of nucleic bases in octanol-water system ${ }^{12}$ correlated linearly with the capacity factor obtained in the present study with aqueous eluent.

Figures 3 and 4 show the relation between the capacity factor and acetonitrile content on the

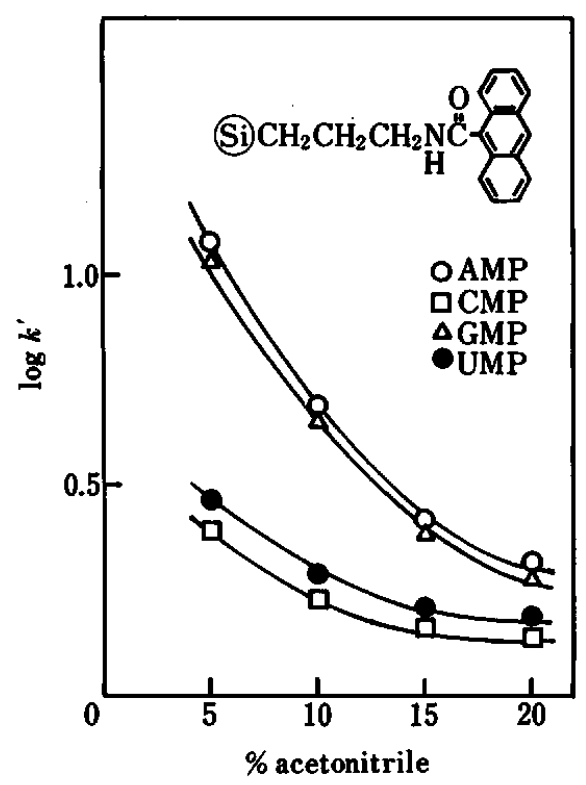

Fig. 3 Chromatography of mononucleotides on anthracenemodified silica gel support. Dependence of capacity factor $\left(k^{\prime}\right)$ on acetonitrile content in mobile phase.

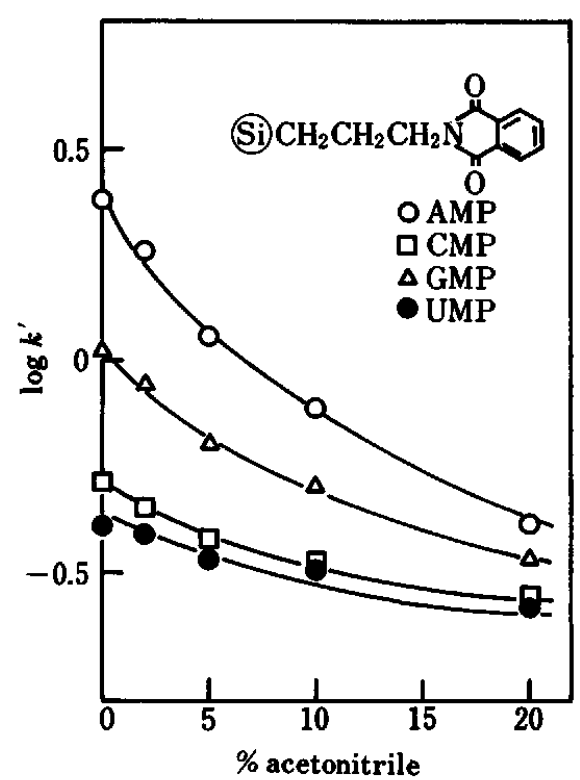

Fig. 4 Chromatography of mononucleotides on phthalimidemodified silica gel support. Dependence of capacity factor $\left(k^{\prime}\right)$ on acetonitrile content in mobile phase. anthracene and phthalimide columns for the four mononucleotides. It is obvious that the addition of acetonitrile causes a general decrease in capacity factor. This behavior indicates that the nature of the present chromatography on the anthracene and phthalimide columns is essentially that of a reversed-phase. However, the nucleotide selectivities are quite different from those obtained with the ODS column. The affinity of the anthracene column to AMP and GMP is more pronounced than those of CMP and UMP. This indicates that the anthracene column discriminates purines (adenine and guanine) from pyrimidines (cytidine and uracil). Therefore, the interaction of anthracene support with mononucleotides is not simply a hydrophobic one, as assessed by water-octanol partition measurement or by capacity factor measurement on an ODS column. Thus, a stacking interaction between anthracene groups and nucleic bases is suggested.

In analogy with the anthracene column, the phthalimide column also shows a tendency to discriminate between purine and pyrimidine bases. It is also worth noting that this column discriminates between AMP and GMP. In Fig. 5 are shown typical chromatograms of four mononucleotides by using phthalimide columns. These indicate that the properties of phthalimide column are intermediate between those of the ODS and the anthracene columns. This can be attributed to the stacking interaction of phthalimide aromatic cycle with nucleic bases being weaker than in the case of anthracene.

Figures 3 and 4 clearly indicate that while the ODS column can not separate mononucleotides under isocratic conditions (at neutral $\mathrm{pH}$ ), anthracene or phthalimide column can do this easily. Stacking of

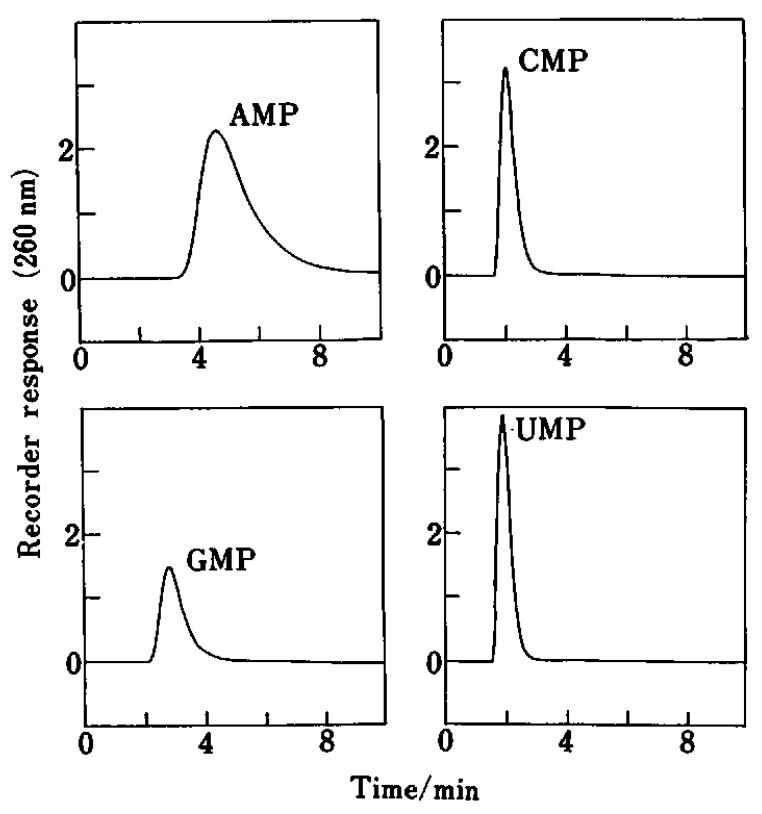

Fig. 5 Chromatograms of mononucleotides (AMP, CMP, GMP, UMP) on phthalimide column: Acetonitrile $0 \%$. 
aromatic compounds is a quite common and widespread phenomenon in organic chemistry; intercalation is just one peculiar case of such phenomena revealed in DNA systems. The observation made above on the chromatographic separation of mononucleotides clearly confirms the potential of explicitly using such molecular interactions for chromatographic (HPLC) separation of nucleotides and related compounds.

\section{Chromatography of oligonucleotides}

Oligonucleotides which are pentadecamer and contain $A, T, C$, and $G$ nucleic bases in various proportions (Fig. 6) were chromatographed. The effect of organic modifier (acetonitrile) was examined in $0.1 \mathrm{M}$ aqueous TEAA buffer eluent system at $\mathrm{pH} 7.01$. Unfortunately, the anthracene column could not be used, since none of the oligonucleotides was eluted from the column even when a high concentration of acetonitrile was used.

Table 1 summarizes the capacity factor of pentadecamers when chromatographed on the ODS column at several acetonitrile concentrations in eluting solvent. Relative values of capacity factor are shown, taking $\mathrm{T}(\mathrm{AT})_{7}$ as a reference compound. In Table 2 are shown similar experimental results when a phthalimide column was used. As easily seen from the comparison of values for ODS (Table 1) and phthalimide (Table 2)

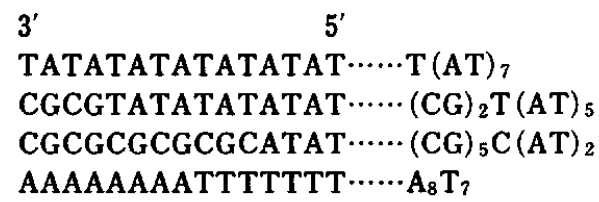

Fig. 6 Structure of pentadecamer oligonucleotides.

Table 1 Effect of acetonitrile content in mobile phase on capacity factor $\left(k^{\prime}\right)$ of oligonucleotides. ODS column.a

\begin{tabular}{|c|c|c|c|}
\hline $\mathrm{MeCN}$ (vol.\%) & Sample & $k^{\prime}$ & $\alpha^{b}$ \\
\hline \multirow[t]{4}{*}{20} & $\mathrm{~T}(\mathrm{AT})_{7}$ & 0.38 & 1.00 \\
\hline & $(\mathrm{CG})_{2} \mathrm{~T}(\mathrm{AT})_{3}$ & 0.40 & 1.05 \\
\hline & $(\mathrm{CG})_{5} \mathrm{C}(\mathrm{AT})_{2}$ & 0.43 & 1.13 \\
\hline & $A_{8} T_{7}$ & 0.43 & 1.13 \\
\hline \multirow[t]{4}{*}{15} & $\mathrm{~T}(\mathbf{A T})_{\eta}$ & 0.82 & 1.00 \\
\hline & $(\mathrm{CG})_{2} \mathrm{~T}(\mathrm{AT})_{\mathbf{s}}$ & 0.68 & 0.82 \\
\hline & $(\mathrm{CG})_{5} \mathrm{C}(\mathrm{AT})_{2}$ & 0.99 & 1.20 \\
\hline & $A_{8} T_{7}$ & 1.06 & 1.29 \\
\hline \multirow[t]{4}{*}{13} & $\mathrm{~T}(\mathbf{A T})_{7}$ & 1.81 & 1.00 \\
\hline & $(\mathrm{CG})_{2} \mathrm{~T}(\mathrm{AT})_{\mathrm{s}}$ & 1.19 & 0.66 \\
\hline & $(\mathrm{CG})_{3} \mathrm{C}(\mathrm{AT})_{2}$ & 2.16 & 1.19 \\
\hline & $A_{8} T_{7}$ & 2.97 & 1.64 \\
\hline
\end{tabular}

a. Mobile phase, TEAA buffer; $\mathrm{pH}=7.01$; flow rate, $0.5 \mathrm{ml}$ $\min ^{-1}$.

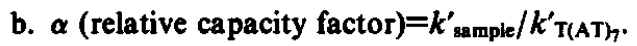

columns, the retention behavior on the two columns are quite different. This again indicates that, though the two columns are operated on the same reversedphase mode (decrease in capacity factor on increasing the acetonitrile content), the nature of the interaction between analyte and support is different. The interaction involved in phthalimide support is not simply hydrophobic, but probably involves stacking or intercalating interactions between phthalimide groups and nucleic bases.

The differences in interaction between the ODS and phthalimide supports with oligonucleotides are most clearly seen by plotting the capacity factor as a function of a number of AT and TA dimer units within pentadecamer oligonucleotides. This is indicated in Fig. 7, where a fraction rather than a number of the dimer units is plotted on the abscissa. ${ }^{13}$ As the fraction AT+TA increases, the capacity factor for phthalimide support increases in a linear fashion. On the other hand, a concave variation is observed for ODS support. Though the data points of $\mathrm{A}_{8} \mathrm{~T}_{7}$ are eliminated ${ }^{14}$ from this profile description, the difference between the ODS and phthalimide supports seems meaningful. It

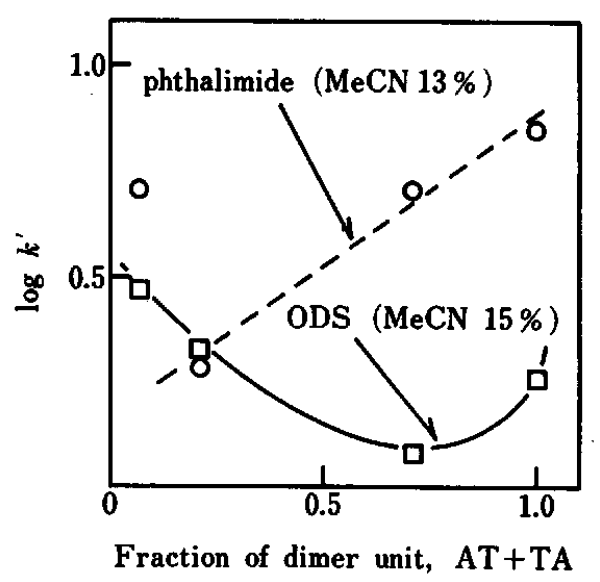

Fig. 7 Dependence of capacity factor $\left(k^{\prime}\right)$ on content of dimer units in pentadecamer oligonucleotides.

Table 2 Effect of acetonitrile content in mobile phase on capacity factor $\left(k^{\prime}\right)$ of oligonucleotides. Phthalimide column. ${ }^{\mathrm{a}}$

\begin{tabular}{|c|c|c|c|}
\hline MeCN (vol.\%) & Sample & $k^{\prime}$ & $\alpha$ \\
\hline \multirow[t]{4}{*}{20} & $\mathbf{T}(\mathbf{A T})_{7}$ & 0.83 & 1.00 \\
\hline & $(\mathrm{CG})_{2} \mathrm{~T}(\mathrm{AT})_{s}$ & 0.75 & 0.90 \\
\hline & $(\mathrm{CG})_{5} \mathrm{C}(\mathrm{AT})_{2}$ & 0.56 & 0.67 \\
\hline & $\mathbf{A}_{8} \mathbf{T}_{7}$ & 0.79 & 0.95 \\
\hline \multirow[t]{4}{*}{15} & $\mathrm{~T}(\mathbf{A T})_{7}$ & 7.19 & 1.00 \\
\hline & $(\mathrm{CG})_{2} \mathrm{~T}(\mathrm{AT})_{5}$ & 5.12 & 0.71 \\
\hline & $(\mathrm{CG})_{5} \mathrm{C}(\mathrm{AT})_{2}$ & 1.94 & 0.27 \\
\hline & $\mathbf{A}_{8} \mathbf{T}_{7}$ & 5.09 & 0.71 \\
\hline
\end{tabular}

a. For conditions and definitions, see Table 1. 


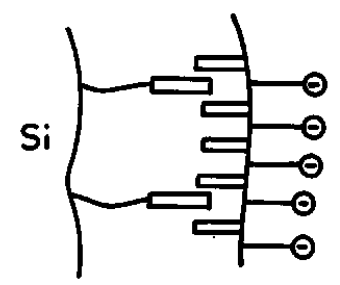

A

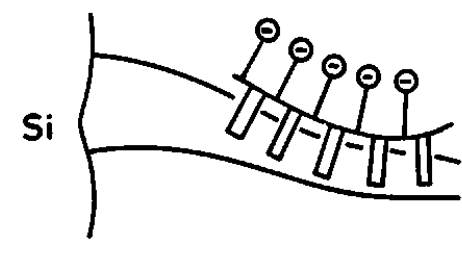

B
Fig. 8 Schematic presentation of interaction between modifiedsilica gel support and oligonucleotide: A, phthalimide support; B, ODS support.

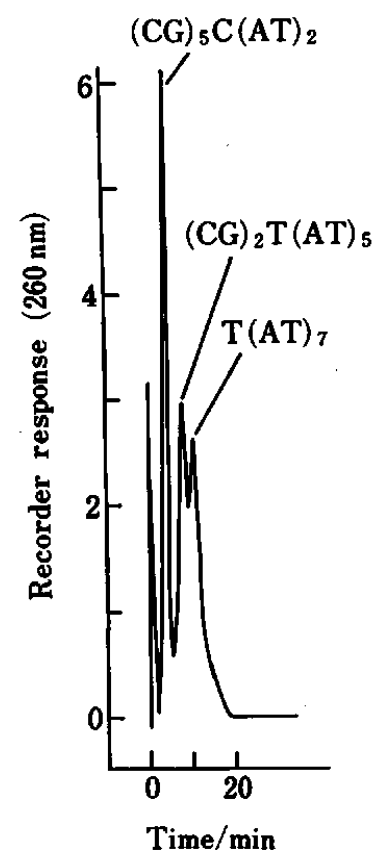

Fig. 9 Chromatogram of oligonucleotides on phthalimide column: Acetonitrile $13 \%$.

would be proper to describe this difference as follows: the ODS support measures the hydrophobicity of oligonucleotides in a general fashion by hydrocarbon chains, whereas the phthalimide support measures the hydrophobicity by aromatic cycles in rather a special fashion through stacking interactions between phthalimide and nucleic bases of oligonucleotides (Fig. 8).

In Figs. 9 and 10 are shown typical chromatograms for the separation of pentadecamers. The estimated number of theoretical plates is about $30 / \mathrm{cm}$, which is lower than that of the ODS column $(303 / \mathrm{cm})$. The ODS column was a commercial one, and the preparation conditions are optimized. On the other hand, the present phthalimide column was prepared just for a preliminary examination. However, several points of possible importance in the development of intercalator-based, reversed-phase chromatographic supports will be considered below.

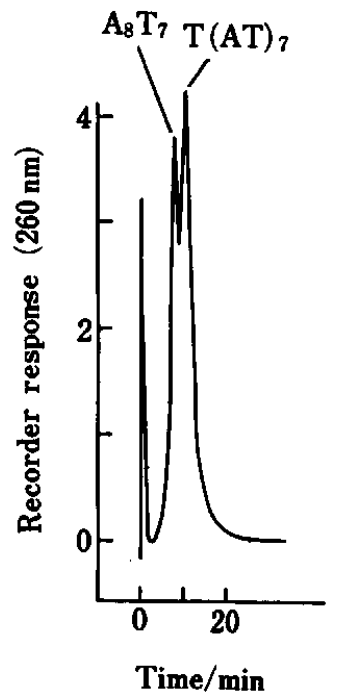

Fig. 10 Chromatogram of oligonucleotides on phthalimide column: Acetonitrile 13\%.

As mentioned before, pentadecamer oligonucleotides were not successfully eluted from the anthracene column. It was not clear whether the oligonucleotides are irreversibly retained on the support or are eluted, but in a very broad band. The residual aminopropyl groups can act as anion exchange sites. However, that they make the primary contribution (through anion exchange) to the unusually strong retention of pentadecamers is doubtful, since a relatively high concentration of buffer salt (0.1 M TEAA) is used in the eluting solution. A hydrophobic, stacking interaction seems to be the major contributing factor.

Now, the extent of stacking interactions can vary from site to site on the support gel beads, though they are prepared with the same chemical treatment. High local concentrations of phthalimide or anthracene groups can give some sites a strong retaining ability, while the sites with low density of the aromatic groups exhibit only a low retention ability. In other words, some sites can behave simply as mono-dentate ligands, while other sites may behave as bidentate or tridentate ligands against the eluting oligonucleotides (Fig. 8). Such situations are quite different from those of ODS support, where the retention of hydrophobic analyte by long-chain hydrocarbons does not presume any special structural requirement (like stacking interactions). In this sense, the surfaces of aromatics-modified support can become much more inhomogeneous than those of ODS in the chromatography of oligonucleotides. This is presumably one of the reasons for the low number of theoretical plates of the phthalimide column. To overcome this difficulty, as well as to avoid an excessive stacking ability inferred by the anthracene column, it may be wise to introduce rather small aromatics on the silica gel surface via flexible and hydrophilic connectors. Such a study is now in progress. 
In conclusion, an intercalator-based HPLC support offers a new possibility in separation of oligonucleotides. The separation is based essentially on a reversed mode, but the mode contains a substantial contribution from stacking interaction between the support-anchored intercalators and nucleic bases in oligonucleotides. For this type of chromatography to attain practical importance, it is still necessary to develop: 1., intercalators which effectively discriminate nucleotide dimer units but do not possess too high an association ability with oligonucleotides, and 2., techniques to modify silica gel support with minimum inhomogeneity using such intercalators.

The authors thank Dr. Y. Kato (Tosoh Co.) for packing chromatographic columns. Thanks are also due to Dojindo Laboratories for their allowing us to use a DNA synthesizer. This work was partially supported by a Grant-in-Aid for Scientific Research from the Ministry of Education, Science and Culture.

\section{References}

1. H. Schott and H. Eckstein, J. Chromatogr., 296, 363 (1984).

2. I. Gillam, S. Millward, D. Blew, M. von Tigerstom, E. Wimmer and G. M. Tener, Biochemistry, 6, 3043 (1967).

3. R. K. Patient, S. C. Hardies, J. E. Larson, R. B. Inman, L. E. Maquat and R. D. Wells, J. Biol. Chem., 254, 5548 (1979).
4. R. Bischoff and L. W. McLaughlin, J. Chromatogr., 296, 329 (1984).

5. R. Bischoff and L. W. McLaughlin, J. Chromatogr., 270, 117 (1983).

6. L. S. Lerman, J. Mol. Biol., 3, 18 (1961).

7. S. Ichimura, M. Zama and H. Fujita, Biochim. Biophys. Acta, 240, 485 (1971).

8. K. Dohtsu, Y.,Katayama and M. Takagi, Chem. Lett., 1986, 1691.

9. S. Takenaka, K. Dohtsu, N. Nakashima and M. Takagi, Anal. Sci., 3, 557 (1987).

10. W. Müller and D. M. Crothers, Eur. J. Biochem., 54, 267 (1975).

11. B. Gaugain, J. Markovits, J-B. L. Pecq and B. P. Roques, Biochemistry, 20, 3035 (1981).

12. A. Leo, C. Hansch and D. Elkins, Chem. Rev., 71, 525 (1971).

13. As an example of calculating the fraction of dimer unit $\mathrm{AT}+\mathrm{TA},(\mathrm{CG})_{5}(\mathrm{AT})_{2}$ contains totally fourteen dimer units, within which there are two AT units and one TA unit. The fraction of AT and TA units is then $(2+1) / 14=0.21$.

14. It is possible that $A_{8} T_{7}$ assumes a somewhat different conformation in solution from other oligonucleotides, and this could be a cause of the discrepancies in the chromatographic behavior of $A_{8} T_{7}$. The presence of nonalternating AT sequence, i.e., base sequences of the type $A_{m} T_{n}$, is suggested to be the cause of a peculiar bending in the tertiary structure of DNA. (H.-S. Koo, H.-M. Wu, D. M. Crothers, Nature[London], 320, 501 (1986).) 